\title{
OPTIMIZING DOUBLE-SIDEBAND SIS QUASIPARTICLE MIXERS
}

\author{
David P. Woody \\ Owens Valley Radio Observatory \\ California Institute of Technology \\ Big Pine, CA 93513
}

and

Michael J. Wengler

Electrical Engineering

University of Rochester

Rochester, NY 14627

\section{Abstract}

Calculations based upon the quantum theory of mixing in single-particle tunnel junctions show that there is a fairly simple strategy for optimizing the performance of double-sideband SIS quasiparticle mixers. The best mixer noise temperature is obtained when the signal source is matched to the LO admittance of the junction. This applies over a very wide range of $\mathrm{LO}$ and $\mathrm{DC}$ bias conditions.

\section{Introduction}

Superconductor-Insulator-Superconductor quasiparticle tunnel junction mixers have proven to be very interesting and useful devices. They exhibit both the phenomena of classical resistive or diode mixers and the quantum behavior of photodector-type mixers. They also provide the lowest noise temperature heterodyne receivers for radio astronomy in the millimeter and submillimeter frequency range. The development of SIS mixers has benefitted greatly from the detailed quantum theory of mixing in single-particle tunnel junctions developed by Tucker [1]. This theory predicted exciting new performance levels for millimeter wavelength mixers, such as quantum limited noise temperatures and conversion gain. An extensive discussion of this theory and review of its experimental verification has been given by Tucker and Feldman [2]. This theory is presented in terms of the standard Y-matrix formalism for mixers [3] plus the corresponding noise current correlation matrix. As such, it's predictions are based upon the details of the RF and IF circuit in which the SIS junction is embedded.

The simplest realizable mixer is a three port device (signal, image, and IF ports) and is described by a $3 \times 3$ matrix whose complex elements depend upon both the DC and LO bias conditions. The extreme nonlinearity of high quality SIS tunnel junctions and the quantum nature of their interaction with radiation leads to a very rich set of performance predictions depending upon the terminations at the various mixer ports and the $\mathrm{LO}$ and DC bias conditions. At frequencies were you can reasonably expect to build or at least characterize the embedding circuit parameters the full $\mathrm{Y}$ matrix formalism can be used to optimize the mixer performance for a particular task. D'Addario has presented the noise parameters for single-sideband SIS mixers [4]. In this case the image port termination is fixed and the extensive formalism for describing the gain and noise of 2-port devices can be applied.

As the operating frequencies increase into the submillimeter wavelength range, the circuit parameters are much harder to pin down and it is useful to develop general design rules for optimizing the mixer performance. Most submillimeter wavelength mixers will have the same signal, image, and LO admittances because of the limited Q's available for the RF circuitry and the low IF frequencies used. This combined with the fact that SIS tunnel junctions are inherently very broadband devices imply that most submillimeter wavelength mixers will be double-sideband devices.

This paper uses computer calculations based upon Tucker's theory [1] plus the inclusion of vacuum fluctuations at the signal and image ports to determine the source admittance which minimizes the noise temperature of double-sideband mixers over a wide range of $\mathrm{LO}$ and $\mathrm{DC}$ bias conditions.

Manuscript received September 24, 1990.

\section{$\underline{\text { Small LO Limit }}$}

The phenomenological theory of mixing [3] can be used to determine the Y-matrix elements for quasiparticle mixers once the device's RF and DC currents are known as a function of the RF and $\mathrm{DC}$ voltages [5]. In this respect SIS quasiparticle mixers are like any other mixer and the conversion efficiency can be calculated in terms of the responsivity using equ. 79 p. 133 of ref. [3]. The responsivity can be determined by the amplitude modulation technique which involves measuring the increment in DC current for a small increment in the LO power. When presented in terms of electrons per photon, the responsivity is the same as the quantum efficiency used to characterize photodetectors.

The noise in the device is a more complicated problem. A complete calculation of the noise requires the current correlation matrix which describes the correlation of the current noise between the various ports. In addition the vacuum fluctuations necessarily present at all of the ports must also be included.

The calculations of the noise properties greatly simplify in the limit of small LO power. The LO bias is given in terms of the dimensionless LO voltage across the junction, $\alpha=\mathrm{eV}_{10} / \mathrm{hf}$, where $h$ is Planck's constant and $\mathrm{f}$ the LO frequency. It can be shown that in the limit of small $\alpha$ the mean square current noise at the IF port is accurately given by the shot noise formula $\mathrm{Iif}^{2}=2 \mathrm{eI} d \mathrm{~d}$, where $\mathrm{Idc}$ is the net DC current including the rectified- or photo-current produced by the $L O$, independent of the source admittance, $Y_{S}$. It has also been shown that high quality tunnel junctions (very little leakage current below the gap voltage, $\mathrm{V}_{\mathrm{g}}$, in the absence of $\mathrm{LO}$ ) biased on the first step below the gap voltage behave like photodiodes, each absorbed photon produces one electron in the DC circuit [6]. Although the small signal admittance which the junction presents at the signal port depends upon the termination of the image and IF ports and can vary wildly, it is the large signal admittance, Ylo, of the junction to which you must couple the signal radiation if you want to achieve the minimum mixer noise temperature [7].

This result is consistent with saying that the shot noise seen in the DC current is simply the result of mixing of the vacuum fluctuations with the coherent $\mathrm{LO}$ field. The vacuum fluctuations are present both in the source and in the "internal modes" of the device so that the current noise at the IF port is independent of how well the source is coupled to the junction. The mixer noise temperature (expressed as the number of photons yielding a signal to noise of one) for high quality junctions is just the inverse of the coupling efficiency between $Y_{S}$ and $Y_{10}$. This is the same as the result obtained for photodetector-type mixers [8].

\section{Finite LO Level}

The situation becomes more complicated when the LO power is increased. As $\alpha$ approaches and exceeds one the detailed noise calculations produce a very rich set of results, especially when you bias the junction at other than the first photon step below the gap. Figure 1 is a projection plot of $I_{d c}$ as a function of both the DC bias voltage, $V_{d c}$, and $\alpha^{2}$ for a high quality SIS tunnel junction. The DC voltage has been normalized to $\Delta / \mathrm{e}$. The LO frequency used for the calculation in Fig. 1 is $\mathrm{f}=0.5 \Delta \mathrm{h}$. The initial large responsivity for $\alpha<1$ followed by a saturation in the responsivity for 


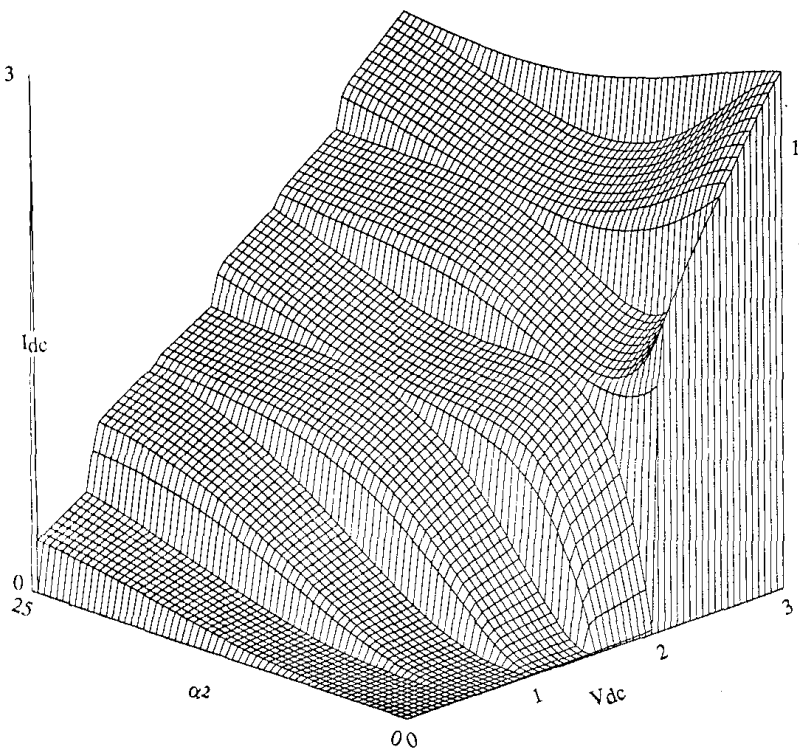

Figure 1. $I_{\mathrm{dc}}$ plotted as a function $\mathrm{V}_{\mathrm{dc}}$ and the LO level for a high quality SIS tunnel junction. $V_{d c}$ has been normalized to $\Delta / c$ and the LO level is given in terms of the square of normalized LO voltage across the junction, $\mathrm{a}^{2}=(\mathrm{eV} / \mathrm{o} / \mathrm{hf})^{2}$. The junction normal state conductance has been normalized to one.

\section{$\alpha>1$ when biased on the first step below $V_{g}$ is clearly seen.}

The noise seen at the IF port depends upon the terminations at the signal and image ports. In Fig. 2 we show the factor by which the IF current noise is suppressed below shot noise when the signal and image ports are terminated by admittances equal to $\mathrm{Y}_{\mathrm{opt}}$ defined below. The decrease in current seen above $V_{g}$ in Fig. 1 has been treated as the conduction of positively charged carriers and thus as an increase in the number of carriers. This plot shows a lot of structure in the deviation of the current noise from simple shot noise in $I_{d c}$. Most of the deviation is in the form of suppressed noise. It is tempting to explain this suppressed noise as a result of the decreased responsivity causing the vacuum fluctuations to be less efficiently converted to output current noise. This simple explanation doesn't work, especially at very large LO levels (beyond the range plotted in Fig. 2) where the responsivity goes to zero but the output noise exceeds the shot noise value.

This would lead one to suspect that there will not be a simple strategy for optimizing the performance of double sideband SIS mixers. It should be noted that the mixer noise temperature is independent of the termination at the IF port, although the achieved conversion efficiency and the receiver noise temperature does depend upon this termination. Figure 3 shows the best mixer noise temperature as a function of the DC and LO bias voltages. The source admittance for the double sideband mixer has been optimized at each bias point to produce the lowest mixer noise temperature. This projection plot shows $h f / \mathrm{kT}_{\mathrm{ssb}}$ so that peaks correspond to good mixer noise temperatures. Generally the best noise temperatures correspond to the bias conditions yielding the largest suppression of the output noise. Interestingly, near quantum limited performance can be achieved as far as three photon steps below $\mathrm{V}_{\mathrm{g}}$ and within a factor of two of the quantum limit is achieved on the first step above $V_{g}$.

The most useful result of this calculation is the determination of the optimum source admittance, $Y_{\text {opt }}$, as a function of the bias conditions. The real part of $Y_{\text {opt }}$ is plotted in Fig, 4. This plot is much less dramatic, the optimum source admittance very closely tracks the well behaved complex conjugate of Ylo. In fact the performance achieved by matching the signal and image ports to $Y_{l}$ differs from the optimum noise temperature

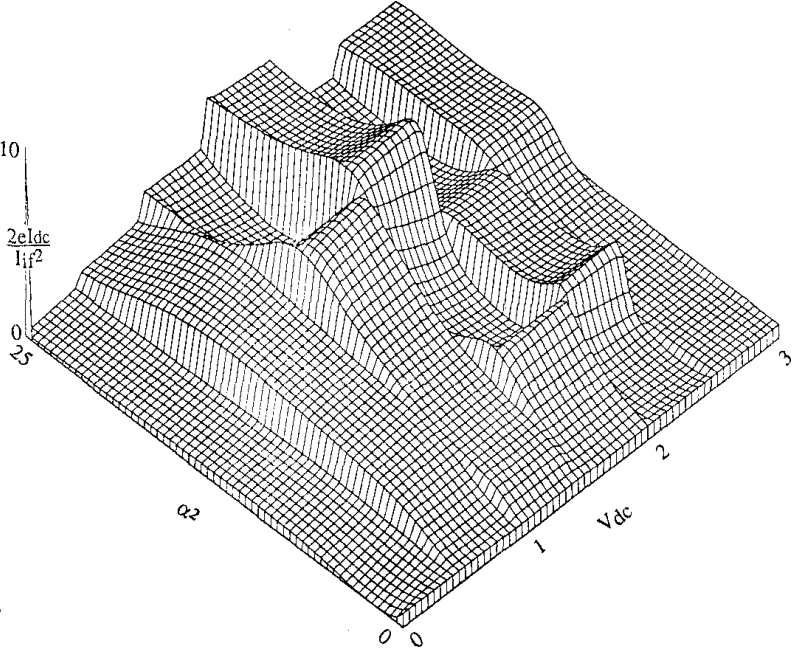

Figure 2. The suppression factor for the noise at the IF port in comparison to shot noise when $Y_{S}$ is equal to $Y_{\text {opt }}$ defined below. The bias parameters are the same as for Fig. 1.

by less than $20 \%$ for most bias conditions and by less than $10 \%$ in the regions where the best mixer performance is achieved.

The best mixer noise temperatures are achieved at source admittances which yield only modest available gains. The available gain at the bias points giving near quantum limited performance is only $3 \mathrm{~dB}$ and the IF conductance to which you want to match the IF amplifier varies from $G_{n}$ to a few times less than this conductance.

The receiver noise temperature, $T_{\text {rec }}$, depends upon the IF amplifier's noise temperature, $T_{i f}$, and the conversion efficiency in addition to the mixer noise temperature. The easiest way to determine the relative contribution of the IF amplifier to the receiver noise is to compare $\mathrm{T}_{\text {if }}$ to the diode noise temperature, $\mathrm{T}_{\text {diode }}=\mathrm{I}_{\mathrm{if}} / 2 / 4 \mathrm{kG}$ if. Diode noise temperatures in the regions yielding the best mixer noise temperatures are in the range $20-50 \mathrm{~K}$. This is much larger than the best available cooled HEMT IF amplifiers which have noise temperatures of $\sim 1 \mathrm{~K}$ per $\mathrm{GHz}$ of operating frequency. Thus receiver performance can be effectively optimized under the same conditions which give the minimum mixer noise temperature.

\section{Discussion}

We have explored the calculated performance for many different junction IV curves and over operating frequencies ranging from $.05 \Delta / \mathrm{h}$ to $\Delta / \mathrm{h}$. They all yield $\mathrm{Y}_{\mathrm{opt}}$ very close to $\mathrm{Y}_{\mathrm{lo}}{ }^{*}$. and have $T_{\text {diode }}$ at the best bias conditions well above $10 \mathrm{~K}$. As expected the highest quality IV curves (least leakage current below $V_{g}$ ) produced the lowest noise mixers and exhibit large suppression of the shot noise. The junctions with significant leakage current below $\mathrm{Vg}$ or operating at relatively low frequencies behave more like classical diode mixers with $\mathrm{I}_{\text {if }}{ }^{2}$ given more closely by the shot noise formula.

The task of optimizing the receiver using a given junction then reduces to building an RF circuit which maximizes the coupling of the LO to the junction. If the circuit has a tuner in it, then it is simply a matter of adjusting the tuner to give the maximum Idc for a given applied LO power. This differs from the common procedure of maximizing the conversion gain. If the RF circuit is fixed, you may want to try biasing over a range of several photon steps and a wide range of LO levels to find where $\mathrm{Y}_{10}$ couples efficiently to the already fixed $Y_{S}$. If the RF circuit admittance is not well known, it may be necessary to try junctions having a range of $G_{n}$ to come up with a good $Y_{S}$ to $Y_{l o}$ match. The junction capacitance is considered to be part of the external RF circuit and must be tuned out to achieve a good $\mathrm{Y}_{\mathrm{S}}$ to $\mathrm{Y}_{\mathrm{lo}}$ coupling. 


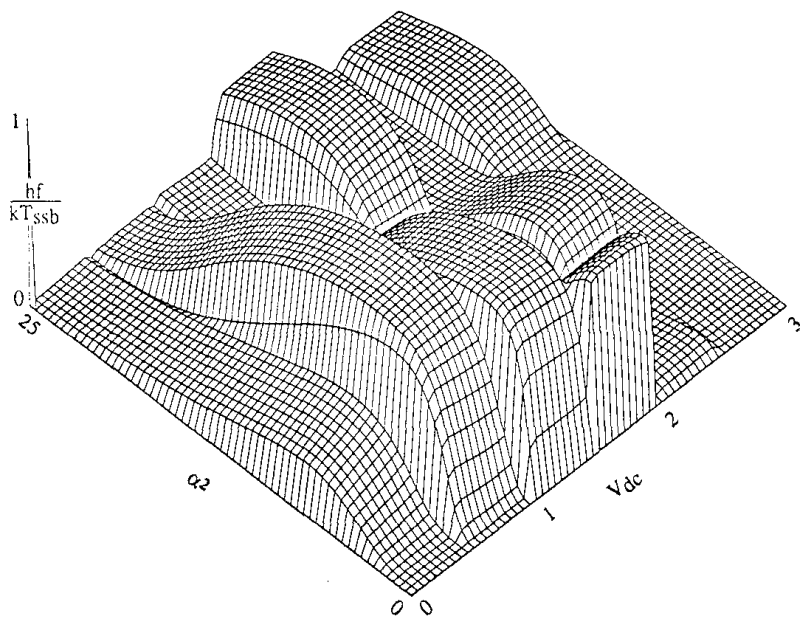

Figure 3. Inverse of the optimized single-sideband mixer noise temperature and normalized to the quantum limit $\mathrm{hf} / \mathrm{k}$. The mixer was optimized by finding the best source admittance as a function of the LO and DC bias.

These calculations support the contention that it is the energy dissipation in the device which is important in determining the noise performance and not the small signal admittance or the power gain. This appears to be another demonstration of the Callen and Welton fluctuation-dissipation theorem which tells us that its is the dissipation of energy which is responsible for the noise generation in a wide range of devices [8] and it is this energy dissipation mechanism to which you want to couple your signal if you want to minimize the noise.

\section{$\underline{\text { References }}$}

1.J. R. Tucker, "Quantum Limited Detection in Tunnel Junction Mixers", IEEE J. Quantum Electron., QE-15, 1234-1258, 1979.

2. J. R. Tucker and M. J. Feldman, "Quantum Detection at Millimeter Wavelengths", Rev. Modern Phys., 57, 1055-1113, 1985.

3. H. C. Torrey, and C. A. Whitmer, "Crystal Rectifiers", (MIT Radiation Lab. Series, Vol. 15) McGraw-Hill, New York, 1948. pp. 119-135.

4. L. R. D'Addario, "Noise Parameters of SIS Mixers", IEEE Trans. Microwave Theory Tech., MTT-36, 1196-1206, 1988.

5. T. G. Phillips and D. P. Woody, "Millimeter- and Submillimeter-Wave Receivers", Ann. Rev. Astron. Astrophys., 20, 285-321, 1982

6. P. K. Tien and J. P. Gordon, "Multiphoton Process Observed in the Interaction of Microwave Fields with the Tunneling between Superconductor Films", Phys. Rev., 129, 647 . 651, 1963; J. R. Tucker and M. F. Millea, "Photon Detection in Nonlinear Tunneling Devices", Appl. Phys. Lett., 33, 611-613, 1978 .

7. M. J. Wengler and D. P. Woody, "Quantum Noise in Heterodyne Detectors", IEEE J. Quantum Electron., QE-23, 613$623,1987$.

8. A. Yariv, "Introduction to Optical Electronics", New York: Holt, Rinehart and Winston, 1976.

9. H. B. Callen and T. A. Welton, "Irreversibility and Generalized Noise", Phys. Rev., 83, 34-40, 1951.

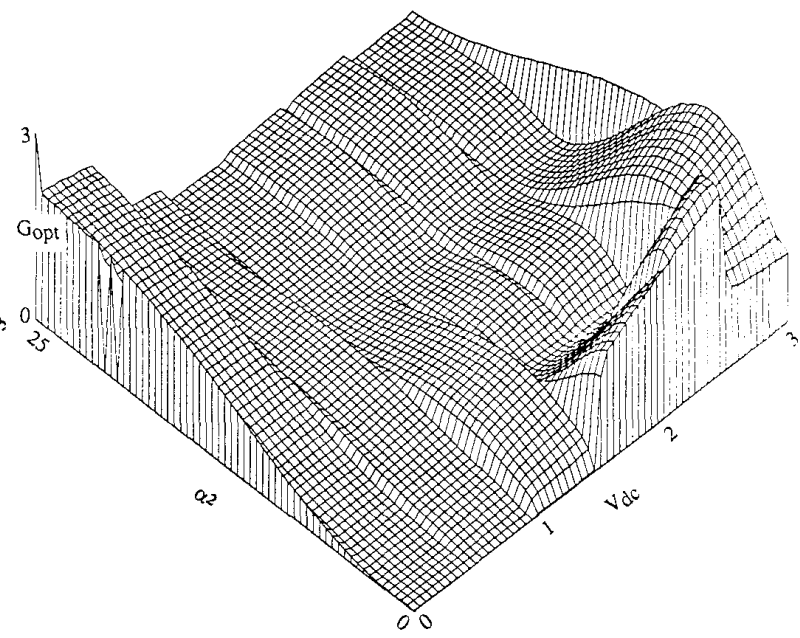

Figure 4. The real part of $\mathrm{Y}_{\mathrm{opt}}$. 